\title{
Narrowly avoided crossings
}

\author{
Michael Wilkinsont \\ Department of Physics, 405-47, California Institute of Technology, Pasadena, CA 91125, \\ USA
}

Received 15 April 1986

\begin{abstract}
In order to create a degeneracy in a quantum mechanical system without symmetries we must vary two parameters in the Hamiltonian. When only one parameter, $\lambda$ say, is varied, there is only a finite closest approach $\Delta E$ of two eigenvalues, and never a crossing. Often the gaps $\Delta E$ in these avoided crossings are much smaller than the mean spacing between the eigenvalues, and it has been conjectured that in this case the gap results from tunnelling through classically forbidden regions of phase space and decreases exponentially as $\hbar \rightarrow 0: \Delta E=A \mathrm{e}^{-S / \hbar}$.

This paper reports the results of numerical calculations on a system with two parameters, $\varepsilon, \lambda$, which is completely integrable when $\varepsilon=0$. It is found that the gaps $\Delta E$ obtained by varying $\lambda$ decrease exponentially as $h \rightarrow 0$, consistent with the tunnelling conjecture. When $\varepsilon=0, \Delta E=0$ because the system is completely integrable. As $\varepsilon \rightarrow 0$, the gaps do not vanish because the prefactor $A$ vanishes; instead it is found that $S$ diverges logarithmically. Also, keeping $\hbar$ fixed, the gaps are of size $\Delta E=\mathrm{O}\left(\varepsilon^{\nu}\right)$, where $\nu$ is usually very close to an integer. Theoretical arguments are presented which explain this result.
\end{abstract}

\section{Introduction}

Consider a quantum mechanical system with a Hamiltonian $\hat{H}$ which depends on a parameter $\lambda$. If $\hat{H}$ has a discrete spectrum, we can plot the eigenvalues $E_{j}$ of $\hat{H}$ as a function of $\lambda$. Naively we might expect to see the curves $E_{j}(\lambda)$ cross each other, as illustrated in figure $1(a)$. This is in conflict with a result of von Neumann and Wigner

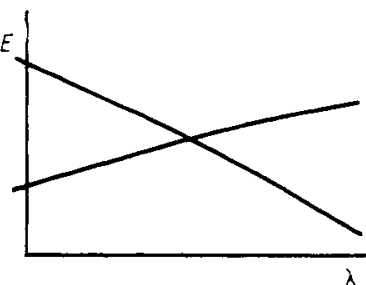

(a)

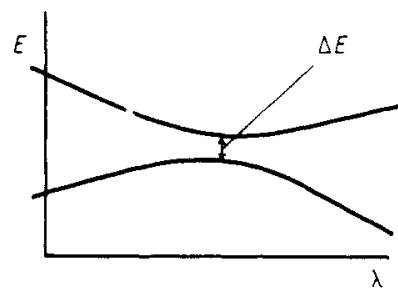

(b)

Figure 1. By varying a parameter $\lambda$ in the Hamiltonian, we might expect to be able to make energy levels degenerate $(a)$. In fact this does not happen for Hamiltonians without symmetry, and instead we observe an avoided crossing $(b)$. Often the gaps $\Delta E$ in these avoided crossings are orders of magnitude smaller than the mean separation of energy levels.

† Address after 1 September 1986: Department of Physics and Applied Physics, John Anderson Building, University of Strathclyde, Glasgow G4 0NG, UK. 
(1929), who proved that in systems without any symmetry we have to vary two parameters in order to create a degeneracy. When only one parameter is varied, there is a finite closest approach $\Delta E_{j}$ of the eigenvalues $E_{j}$ and $E_{j+1}$ rather than a degeneracy. This avoided crossing is illustrated in figure $1(b)$.

Often the gaps $\Delta E_{j}$ of the avoided crossings are orders of magnitude smaller than the mean value of the level separation, $E_{j+1}-E_{j}$. This can be explained by the following argument. Assume that the classical Hamiltonian which corresponds to the quantum system has trajectories which are confined to tori in phase space. The KAM theorem suggests that most systems have some of these phase space tori. The quantum energy levels associated with phase space tori can be calculated by the Einstein-BrillouinKeller (EBK) quantisation scheme: if $H(\boldsymbol{I})$ is the Hamiltonian as a function of the action variables $I$ then the quantum eigenvalues are given by

$$
E_{n}=H\left(I_{n}\right) \quad I_{n}=2 \pi\left(n+\frac{1}{4} \alpha\right) \hbar
$$

where the vector $\boldsymbol{\alpha}$ has constant integer elements called Maslov indices (Berry 1983). The EBK quantisation scheme predicts that energy levels can cross, as illustrated in figure $1(a)$, but since it is only an asymptotic scheme, and not exact, we still expect to see the degeneracies split by a finite amount, as in figure $1(b)$. It is possible, however, to write down corrections to the EBK quantisation scheme to any order in $\hbar$, and these corrections will depend only on the properties of a single torus $I$. These corrections cannot therefore be used to calculate $\Delta E$, so that the splitting must vanish faster than any power of $\hbar$ as $h \rightarrow 0$. This explains the very small values of $\Delta E$ which have been observed in numerical experiments, at least when both $E_{j}$ and $E_{j+1}$ are eigenvalues corresponding to phase space tori (Lawton and Child 1981, Davis and Heller 1981).

A possible form for $\Delta E$ as a function of $\hbar$ is

$$
\Delta E=A \mathrm{e}^{-S / \hbar}
$$

where $S$ is a constant and $A$ may have a power law dependence on $\hbar$; this expression is not analytic at $\hbar=0$, and its Taylor expansion about this point is identically zero. It has been conjectured that $\Delta E$ is of this form, and results from tunnelling between the two tori in phase space (Lawton and Child 1981), but this has never been adequately tested by numerical experiments. This paper reports calculations of $\Delta E$ for a fixed pair of tori $\boldsymbol{I}_{1}, \boldsymbol{I}_{2}$, and they are consistent with the tunnelling conjecture (1.2), with $A$ proportional to $\hbar^{3 / 2}$.

This tunnelling effect is very different from most of the familiar examples of quantum mechanical tunnelling, which invole tunnelling through a classically forbidden region between two separate regions of coordinate space. The EBK quantised tori do not intersect in phase space (except at complex values of the coordinates), but their projections onto coordinate space will usually overlap, and there is no spatial separation of the two wavefunctions which participate in the tunnelling process.

The Hamiltonian chosen for this investigation was of the form $H=H_{0}(\lambda)+\varepsilon H_{1}$, where $H_{0}$ is exactly integrable for all $\lambda$, and $\varepsilon H_{1}$ is a perturbation which makes the system quasi-integrable. The quantum Hamiltonian $\hat{H}_{0}(\lambda)$ is also exactly integrable for all $\lambda$, i.e. the constant of motion $K$ can be represented by an operator $\hat{K}$ which commutes with $\hat{H}_{0}(\lambda)$ exactly: $\left[\hat{H}_{0}, \hat{K}\right]=0$. This symmetry of $\hat{H}_{0}(\lambda)$ implies that when $\varepsilon=0$ eigenvalues can cross as $\lambda$ is varied, i.e. $\Delta E=0$. It is natural to suppose that $\Delta E$ vanishes when $\varepsilon=0$ because the prefactor $A$ in (1.2) vanishes. A theoretical analysis suggests that this is not the case, but rather $\Delta E$ vanishes because the tunnelling action $S$ diverges logarithmically as $\varepsilon \rightarrow 0$ (Wilkinson 1986). The action for tunnelling between 
two EBK quantised states was estimated for several values of $\varepsilon$, and the results are consistent with the relation

$$
S=-C \ln \varepsilon
$$

where $C$ is a constant.

Equation (1.3) suggests that if we calculate $\Delta E$ as a function of $\varepsilon$ with $\hbar$ fixed, it will obey a power law in the limit $\varepsilon \rightarrow 0$ :

$$
\Delta E=\alpha \varepsilon^{\nu}
$$

The numerical results are consistent with (1.4) being satisfied with integer values of $\nu$, which are different for different pairs of states. A rule was formulated for determining $\nu$, and the origin of this rule is explained using perturbation theory.

Section 2 will introduce the model system used for these calculations. Section 3 will present the numerical results and $\S 4$ is a conclusion. The origin of the rule for obtaining the integer exponents $\nu$ is explained in an appendix.

\section{The model Hamiltonian}

A set of $N$ interacting spins $\left\{s^{(\alpha)}\right\}$, described by a Hamiltonian $H\left(s^{(1)}, \ldots, s^{(N)}\right)$, obeys the classical equations of motion

$$
\dot{s}_{i}^{(\alpha)}=\varepsilon_{i j k} \frac{\partial H}{\partial s_{j}^{(\alpha)}} s_{k}^{(\alpha)} .
$$

The magnitudes of the spins are conserved, so that the motion is confined to a $2 N$-dimensional manifold which is the product of $N$ spheres. Also, it can be shown that the flow defined by (2.1) preserves a volume element on this manifold, and is therefore equivalent to a Hamiltonian flow in a phase space with $N$ coordinates and $N$ momenta. Spin systems can therefore be used as models for Hamiltonian systems, and in semiclassical quantum mechanics there is a considerable advantage in doing this. Because the phase space is a compact manifold, it is possible to use a complete finite-dimensional basis to represent the state of the corresponding quantum system, and the Hamiltonian can therefore be represented exactly by a finite-dimensional matrix. Because it is not necessary to use a truncated basis set, all the eigenvalues obtained by diagonalising the Hamiltonian matrix are meaningful, and their accuracy is limited only by that of the computer program (Feingold and Peres 1983). For these reasons a spin system was used as the model for this study.

The system chosen has an integrable component $\hat{H}_{0}(\lambda)$ and a non-integrable perturbation: $\hat{H}=\hat{H}_{0}(\lambda)+\varepsilon \hat{H}_{1}$. The integrable component was

$$
\hat{H}_{0}(\lambda)=\frac{1}{s^{2}}\left[\hat{s}_{1} \cdot \hat{s}_{2}+\lambda s\left(\hat{s}_{1 z}+\hat{s}_{2 z}\right)\right]
$$

where $\hat{s}_{1}, \hat{s}_{2}$ are two spins of spin $j$ and $s=\sqrt{j(j+1)}$. This Hamiltonian represents two spins interacting isotropically and with an external magnetic field of strength $\lambda$. The factors involving $s$ are included so that all terms are of order unity in the classical limit, $j \rightarrow \infty$. For most of the numerical work the perturbing Hamiltonian was

$$
\hat{H}_{1}=\hat{s}_{1 x} \hat{s}_{2 x}
$$


which corresponds to making the interaction between the spins anisotropic. For some of the trials a different perturbation was used:

$$
\hat{H}_{1}^{\prime}=s\left(\hat{s}_{1 x}-\hat{s}_{2 x}\right)
$$

which makes the external field applied to each spin point in a different direction. For both choices of the perturbation it was verified by integrating (2.1) that the corresponding classical motion was of the KAM type.

The unperturbed Hamiltonian $\hat{H}_{0}(\lambda)$ is exactly integrable quantum mechanically as well as classically; the eigenvalues of (2.2) are

$$
E_{J, M}=\left(1 / s^{2}\right)\left[\frac{1}{2} J(J+1)+\lambda s M-s^{2}\right]
$$

where $J$ and $M$ vary by integer increments in the ranges

$$
-2 j \leqslant J \leqslant 2 j \quad-J \leqslant M \leqslant J
$$

(Landau and Lifshitz 1958). Comparing (2.5) with the EBK quantisation condition, we find that the effective Planck's constant in this problem is

$$
2 \pi \hbar=1 / s=[j(j+1)]^{-1 / 2}
$$

that $H(I)$ is

$$
H\left(I_{J}, I_{M}\right)=\frac{1}{2} I_{J}^{2}+\lambda I_{M}-1
$$

and that the Maslov indices associated with $I_{J}, I_{M}$ are 2 and 0 respectively. The angle variables $\theta_{M}, \theta_{J}$ associated with the actions $I_{M}, I_{J}$ describe respectively the precession of the spins about their resultant and the precession of this resultant about the externally applied field.

It is important for the theoretical discussion in the appendix to express the perturbations $H_{1}, H_{1}^{\prime}$ in terms of the action-angle variables. The first step is to express the unit vectors $s_{1}, s_{2}$ in terms of action-angle variables. After some manipulation of rotation matrices, we find $s_{1}=\left(\begin{array}{c}s_{1 x} \\ s_{1 y} \\ s_{1 z}\end{array}\right)=\left(\begin{array}{c}\cos \varphi \sin \chi \cos \theta_{M} \cos \theta_{J}-\sin \chi \sin \theta_{M} \cos \theta_{J}+\cos \chi \sin \theta \cos \theta_{M} \\ \cos \varphi \sin \chi \sin \theta_{M} \cos \theta_{J}+\sin \chi \cos \theta_{M} \sin \theta_{J}+\cos \chi \sin \theta \sin \theta_{M} \\ -\sin \varphi \sin \chi \cos \theta_{J}+\cos \varphi \cos \chi\end{array}\right)$

where

$$
\cos \varphi=\frac{1}{2} I_{M} \quad \frac{1}{2} I_{J}^{2}-1=\cos 2 \psi .
$$

The expression for $s_{2}$ is the same, except that $\theta_{M}$ is shifted by $\pi$. Using (2.9) we find

$$
\begin{aligned}
H_{1}=\cos ^{2} \chi \sin ^{2} \varphi \cos ^{2} \theta_{M}+2 \sin ^{2} \chi \cos \varphi \cos \theta_{M} \sin \theta_{M} \cos ^{2} \theta_{J} \\
-\sin ^{2} \chi \cos ^{2} \varphi \cos ^{2} \theta_{M} \cos ^{2} \theta_{J}-\sin ^{2} \chi \sin ^{2} \theta_{M} \cos ^{2} \theta_{J}
\end{aligned}
$$

and

$H_{1}^{\prime}=2\left(\cos \chi \sin \chi \cos \theta_{M} \cos \theta_{J}-\sin \chi \sin \theta_{M} \cos \theta_{J}+\cos \chi \sin \varphi \cos \theta_{M}\right)$.

Finally, we comment on the symmetries of the model system. When the perturbation $\hat{H}_{1}$ is applied to $\hat{H}_{0}(\lambda)$, there remain two discrete two-fold symmetries: there is symmetry under exchange of the spins, and also a less obvious symmetry: if we express the Hamiltonian in the basis $\left|m_{1}, m_{2}\right\rangle$ of eigenstates of $\hat{s}_{1 z}$ and $\hat{s}_{2 z}$, we find that only states 
with the same parity of $M=m_{1}+m_{2}$ are coupled. Because of these symmetries the Hamiltonian matrix of dimension $(2 j+1)^{2}$ decomposes into four block-diagonal parts of roughly equal size. The eigenvalues in different symmetry classes can cross each other as $\lambda$ is varied; only pairs of eigenvalues within a symmetry class exhibit avoided crossings. The other perturbing Hamiltonian, $H_{1}^{\prime}$, also does not completely remove the symmetries of $\hat{H}_{0}$; in this case the Hamiltonian only has one discrete symmetry, under the operation of simultaneously exchanging and reversing the sign of both spins.

\section{Numerical experiments}

Two series of numerical experiments were performed. One was intended to test the tunnelling conjecture, and examined the dependence of $\Delta E$ on $\hbar$ (i.e. the spin $j$ ) and $\varepsilon$ for a fixed pair of tori, $\boldsymbol{I}_{1}$ and $\boldsymbol{I}_{2}$. The other was an investigation of the perturbation theory of the system, and examined $\Delta E$ as a function of $\varepsilon$ with $\hbar$ fixed, for various pairs of states.

\subsection{Investigation of tunnelling effects}

In these experiments $\Delta E$ was computed for a pair of states with quantum numbers $\boldsymbol{n}_{1}, \boldsymbol{n}_{2}$ proportional to $j$ :

$$
\begin{aligned}
& \boldsymbol{n}_{1}=\left(J_{1}, M_{1}\right)=\left(\frac{1}{2} j, 0\right) \\
& \boldsymbol{n}_{2}=\left(J_{2}, M_{2}\right)=\left(\frac{3}{2} j,-j\right)
\end{aligned}
$$

where $j$, the spin of $s_{1}$ and $s_{2}$, took even integer values from 2 to 16 . This corresponds to looking at $\Delta E$ for a fixed pair of tori $I_{1}, I_{2}$ at various values of $\hbar$.

The results are presented in table 1 . For all values of $\varepsilon$, the position of the avoided crossing in $(E, \lambda)$ space tended toward a well defined limit $\left(E^{*}, \lambda^{*}\right)$ as $j \rightarrow \infty$. This is

Table 1. Values of $\Delta E$ for avoided crossing between states $n_{1}=\left(J_{1}, M_{1}\right)=\left(\frac{1}{2} j, 0\right)$ and $n_{2}=\left(\frac{3}{2} j,-j\right)$ for various even integer values of the spin $j$. For all entries in this table the perturbation was $\varepsilon \hat{s}_{1, x} \hat{s}_{2 x}$. In the limit $j \rightarrow \infty$, the position of the avoided crossing in $(E, \lambda)$ space tends toward $\left(E^{*}, \lambda^{*}\right)$. Most values of $\Delta E$ are much smaller than the mean separation of eigenvalues, $\delta$. Some values of $\Delta E$ are absent because they were too small to be computed reliably (a), or because the avoided crossing was not well separated from other avoided

\begin{tabular}{|c|c|c|c|c|c|c|c|c|c|}
\hline & \multicolumn{8}{|c|}{$\varepsilon$} & \multirow[b]{2}{*}{$\delta$} \\
\hline & 0 & 0.05 & 0.1 & 0.2 & 0.3 & 0.4 & 0.5 & 0.7 & \\
\hline \multirow[t]{6}{*}{$j$} & 0 & 0.00924 & 0.0187 & 0.0382 & 0.0583 & 0.0792 & 0.101 & 0.145 & 0.35 \\
\hline & 0 & 0.000404 & 0.00160 & 0.00609 & 0.0128 & 0.0212 & 0.0307 & 0.0522 & 0.11 \\
\hline & 0 & 0.0000201 & 0.000158 & 0.00119 & 0.00363 & 0.00760 & 0.0129 & 0.0266 & 0.051 \\
\hline & 0 & a & 0.0000168 & 0.000248 & 0.00111 & 0.00300 & 0.00601 & 0.0154 & 0.029 \\
\hline & 0 & $\mathbf{a}$ & a & 0.0000542 & 0.000356 & 0.00124 & 0.00345 & 0.00796 & 0.019 \\
\hline & 0 & $\mathbf{a}$ & a & a & 0.000118 & 0.000487 & 0.00156 & $b$ & 0.014 \\
\hline 14 & 0 & $\mathrm{a}$ & $\mathbf{a}$ & a & 0.0000348 & 0.000230 & 0.000802 & 0.00401 & 0.010 \\
\hline 16 & 0 & $\mathbf{a}$ & $\mathbf{a}$ & $\mathbf{a}$ & 0.0000136 & 0.000103 & 0.000302 & 0.00282 & 0.0078 \\
\hline$E^{*}$ & $-\frac{7}{8}$ & -0.88 & -0.89 & -0.90 & -0.918 & -0.931 & -0.94 & -0.97 & \\
\hline$\lambda^{*}$ & 1 & 1.013 & 1.021 & 1.038 & 1.054 & 1.070 & 1.082 & 1.101 & \\
\hline
\end{tabular}
crossings (b). 
strong evidence that the states correspond to classical tori, and that their energies could be predicted by the EBK quantisation rule. In most cases the gap $\Delta E$ at the avoided crossing is much smaller than the mean separation of energy levels $\delta$ within the symmetry class (which was calculated analytically using the Weyl rule at $\varepsilon=0$ ).

Theoretical arguments suggest that the prefactor $A$ of (1.2) is proportional to $\hbar^{3 / 2}$ (Wilkinson 1986). Accordingly, $\ln \left(\hbar^{-3 / 2} \Delta E\right)$ was plotted against $s=1 / 2 \pi \hbar$ for different values of $\varepsilon$. For values of $\varepsilon$ less than 0.5 a straight line is obtained at large values of $s$, corresponding to $j \geqslant 6$. This supports both the tunnelling conjecture and the prediction that the prefactor is proportional to $\hbar^{3 / 2}$. The slope of this line is $-2 \pi S$, where $S$ is the action for tunnelling between the pair of tori $I_{1}=\left(\frac{1}{2}, 0\right), I_{2}=\left(\frac{3}{2},-1\right)$. For values of $\varepsilon$ greater than or equal to 0.5 , the results are slightly erratic, and do not quite fit a straight line. This could be due to the onset of the breakdown of the phase space tori at large values of the perturbation parameter $\varepsilon$.

Table 2 lists the values of the tunnelling action $S$ deduced from the straight line fits to the data plotted in figure 2. There is no indication that $S$ tends toward a limit as $\varepsilon \rightarrow 0$, and theoretical arguments (Wilkinson 1986) suggest that $S$ should diverge $\operatorname{logarithmically~as~} \varepsilon \rightarrow 0$. Figure 3 is a plot of $S$ against $\ln \varepsilon$, and the data are fitted reasonably well by a straight line, supporting this prediction. The constant of proportionality $C$ in (1.3) was estimated to be $C=7.75 \times 10^{-3}$ from the straight line in figure 3 .

From the data in table 1, we can see that for most of the avoided crossings, the energy $E^{*}$ is shifted from its unperturbed value at $\varepsilon=0$ by an amount which exceeds the mean level spacing $\delta$. This shows that the results describe a regime in which perturbation theory is not applicable, and is therefore further evidence that they are indicative of a tunnelling effect.

Table 2. The tunnelling action, $S$, between the tori $I_{1}=\left(\frac{1}{2}, 0\right), I_{2}=\left(\frac{3}{2},-1\right)$, for various values of $\varepsilon$. The results for $\varepsilon=0.5$ and 0.7 should be considered less reliable, because the data showed some scatter (see figure 2).

\begin{tabular}{ll}
\hline$\varepsilon$ & $S$ \\
\hline 0.2 & 0.093 \\
0.3 & 0.065 \\
0.4 & 0.047 \\
0.5 & 0.034 \\
0.7 & 0.014 \\
\hline
\end{tabular}

\subsection{Investigation of perturbation theory}

In these experiments $\Delta E$ was computed as a function of $\varepsilon$ with $\hbar$ held fixed, for avoided crossings between various pairs of states. Some representative results are given in table 3. The results fit a power law, $\Delta E=a \varepsilon^{\nu}$, and with one exception values of $\nu$ were found to be very close to integers (see figure 4).

The exponent $\nu$ was found for several avoided crossings, and the results are listed in table 4. Most of these results are for the perturbation $\hat{H}_{1}$ given by $(2.3)$, but some results are also included for the perturbation $\hat{H}_{1}^{\prime}(2.4)$. A rule was formulated which relates $\nu$ to the quantum numbers $n_{1}=\left(J_{1}, M_{1}\right)$ and $n_{2}=\left(J_{2}, M_{2}\right)$ of the pair of states 


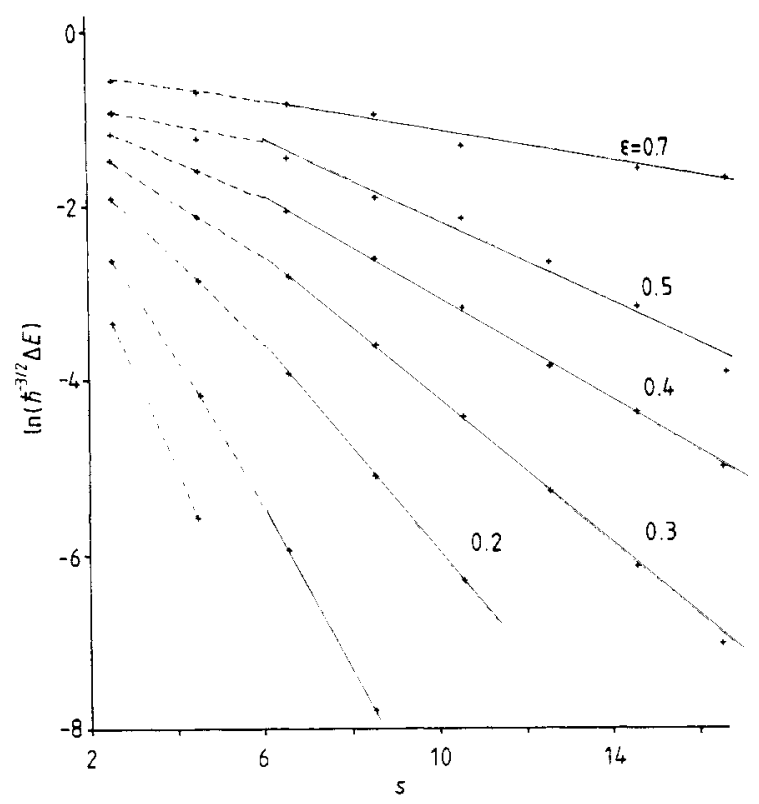

Figure 2. Plot of $\ln \left(\hbar^{-3 / 2} \Delta E\right)$ against $s=\sqrt{j(j+1)}=1 / 2 \pi \hbar$, for various values of $\varepsilon$. The data fit a straight line for $s \geqslant 6$, and the slope of this line is $-2 \pi S$, where $S$ is the tunnelling action. For values of $\varepsilon$ up to 0.4 this fit is to within the error in the data, but for the largest two values of $\varepsilon$ there is some scatter.

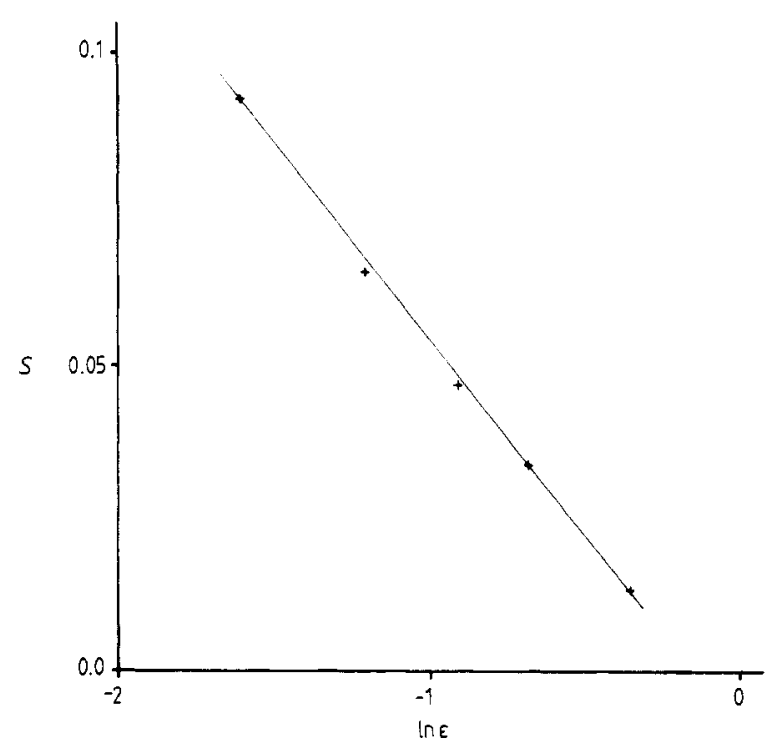

Figure 3. Plot of the tunnelling action, deduced from figure 2, against $\ln \varepsilon$. 
Table 3. Values of $\Delta E$ as a function of $\varepsilon$ for avoided crossings between various pairs of states with quantum numbers $\boldsymbol{n}_{1}=\left(J_{1}, M_{1}\right), \boldsymbol{n}_{2}=\left(J_{2}, M_{2}\right)$. The column labelled $H_{1}$ indicates which perturbation was used: the perturbation $\varepsilon \hat{s}_{1 x} \hat{s}_{2 x}$ is indicated by (a) and $\varepsilon s\left(\hat{s}_{1 x}-\hat{s}_{2 x}\right)$ by (b). The results fit the power law $\Delta E=\alpha \varepsilon^{\nu}$, and usually the value of $\nu$ is very close to an integer.

\begin{tabular}{|c|c|c|c|c|c|c|c|c|c|c|c|c|c|}
\hline \multirow[b]{2}{*}{$j$} & \multirow[b]{2}{*}{$J_{1}$} & \multirow[b]{2}{*}{$M_{1}$} & \multirow[b]{2}{*}{$J_{2}$} & \multirow[b]{2}{*}{$M_{2}$} & \multirow[b]{2}{*}{$H_{1}$} & \multicolumn{8}{|c|}{$\Delta E$} \\
\hline & & & & & & $\varepsilon=0.0125$ & $\varepsilon=0.025$ & $\varepsilon=0.05$ & $\varepsilon=0.075$ & $\varepsilon=0.1$ & $\varepsilon=0.2$ & $\varepsilon=0.4$ & $\nu$ \\
\hline 4 & 2 & 0 & 6 & -4 & $a$ & - & - & 0.000404 & - & 0.00160 & 0.00609 & 0.0212 & 1.96 \\
\hline 4 & 6 & 4 & 8 & 0 & $a$ & - & - & 0.0000078 & - & 0.0000312 & 0.000128 & 0.000538 & 2.04 \\
\hline 4 & 2 & 2 & 6 & -4 & $a$ & - & - & - & - & 0.0000073 & 0.0000928 & 0.001061 & 3.59 \\
\hline 4 & 2 & 0 & 6 & -4 & $b$ & 0.0000111 & 0.000172 & 0.00238 & 0.00916 & - & - & $\ldots$ & 3.88 \\
\hline
\end{tabular}

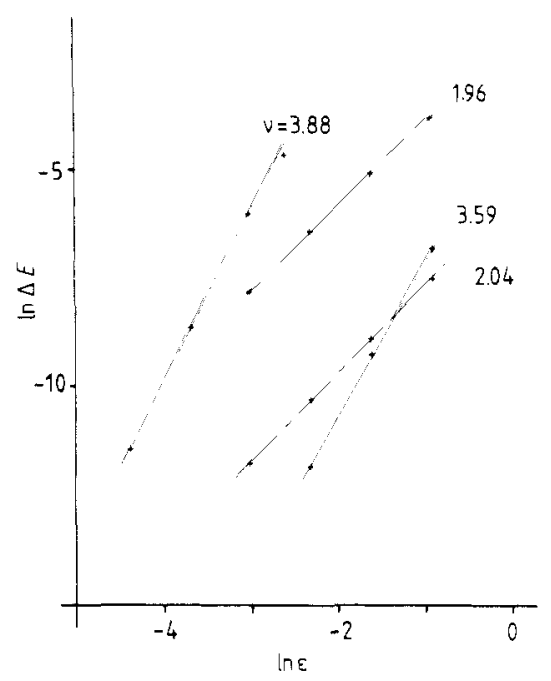

Figure 4. Plot of $\ln \Delta E$ against $\ln \varepsilon$ for the data in table 3 . The slopes $\nu$ of these lines usually have values very close to integers.

Table 4. Observed and predicted values of the exponent $\nu$ for avoided crossings between various pairs of states $n_{1}=\left(J_{1}, M_{1}\right), n_{2}=\left(J_{2}, M_{2}\right)$. The column labelled $H_{1}$ indicates whether the perturbation applied was $\varepsilon \hat{s}_{1, x} \hat{s}_{2 x}($ a $)$ or $\varepsilon s\left(\hat{s}_{1 x}-\hat{s}_{2 x}\right)($ b).

\begin{tabular}{llllllll}
\hline$j$ & $J_{1}$ & $M_{1}$ & $J_{2}$ & $M_{2}$ & $H_{1}$ & $\nu_{\text {obs }}$ & $\nu_{\text {pred }}$ \\
\hline 5 & 3 & 0 & 9 & -4 & $\mathrm{a}$ & 2.90 & 3 \\
4 & 2 & 0 & 6 & -4 & $\mathrm{~b}$ & 3.88 & 4 \\
4 & 2 & 0 & 6 & -4 & $\mathrm{a}$ & 1.96 & 2 \\
6 & 3 & 0 & 9 & -6 & $\mathrm{a}$ & 2.94 & 3 \\
4 & 6 & 4 & 8 & 0 & $\mathrm{a}$ & 2.04 & 2 \\
4 & 2 & 2 & 6 & -4 & $\mathrm{a}$ & 3.59 & 3 \\
4 & 2 & 2 & 6 & -2 & $\mathrm{a}$ & 2.09 & 2 \\
5 & 3 & 0 & 9 & -6 & $\mathrm{a}$ & 2.83 & 3 \\
3 & 1 & 0 & 3 & -2 & $\mathrm{~b}$ & 1.79 & 2 \\
\hline
\end{tabular}


involved in the avoided crossing, and to the functional form of the perturbation. The first step is to express the perturbation $H_{1}$ or $H_{1}^{\prime}$ in action-angle variables; this was done in $\S 2$, and the results are (2.11) and (2.12). The Fourier coefficients of the angle variables can be represented as points on a lattice, $\boldsymbol{k}=\left(k_{f}, k_{M}\right)$, and the non-vanishing Fourier coefficients are considered to link points on this lattice separated by a vector $\boldsymbol{k}$. These linkages are illustrated in figure 5 for the two perturbations considered. The rule for calculating $\nu$ is as follows: we represent the quantum numbers $\boldsymbol{n}_{1}, \boldsymbol{n}_{2}$ as points on a lattice, and find the minimum number of linkages required to connect them. This minimum number is the exponent $\nu$. The use of this construction is illustrated in figure 6. This rule predicts $\nu$ correctly for all of the avoided crossings listed in table 4, except for the sixth entry, where $\nu$ was not close to an integer.

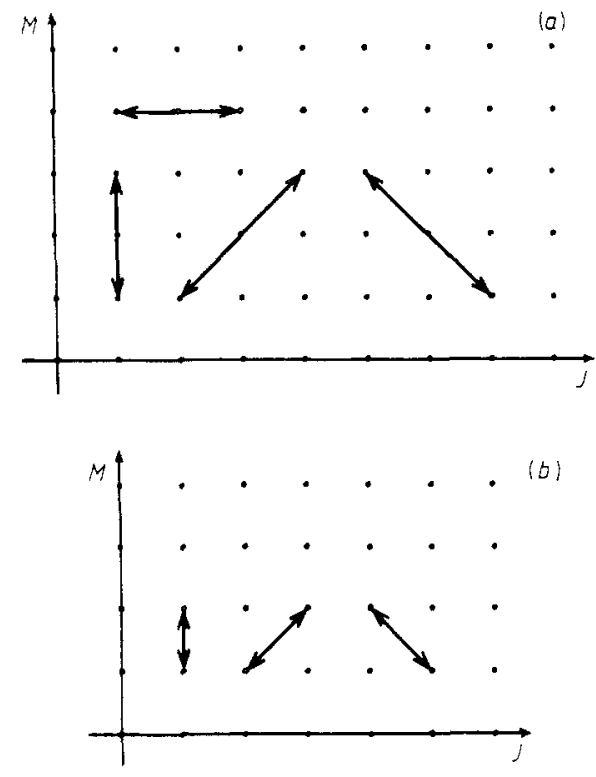

Figure 5. Linkages on the action lattice defined by the perturbation $\hat{H}_{1}:(a) \hat{H}_{1}=\hat{s}_{1 x} \hat{s}_{2 x}$, (b) $\hat{H}_{1}=s\left(\hat{s}_{1 x}-\hat{s}_{2 . x}\right)$.

\section{Conclusion}

The results presented in $\$ 3.1$ generally support the hypothesis that the gaps in avoided crossings between EBK quantised states in KAM systems are due to a tunnelling effect. They also support theoretical predictions (Wilkinson 1986) that the prefactor is proportional to $\hbar^{3 / 2}$ for systems with two degrees of freedom, and that $S$ diverges logarithmically as a parameter is varied which makes the system exactly integrable.

The only aspect of the results which is not fully in accord with these predictions is that for the largest two values of $\varepsilon(0.5$ and 0.7$)$, the data plotted in figure 2 did not fit a straight line exactly, although they show the correct trend. This may be due to the onset of breakdown of the KAM tori for large values of the perturbation parameter $E$, but the results did not show any other signs of this; for instance the position of the avoided crossing still appears to converge to a well defined limit $\left(E^{*}, \lambda^{*}\right)$ as $j \rightarrow \infty$. It 


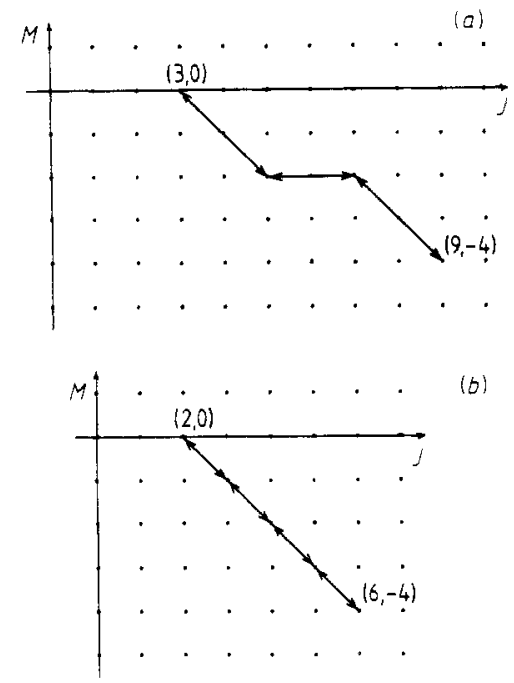

Figure 6. Illustrating the calculation of $\nu$ by counting the minimum number of linkages required to join the states $n_{1}$ and $n_{2}$ on the action lattice. The two examples correspond to $(a)$ the first and $(b)$ the second entry in table 4 .

is quite possible, however, that the tunnelling effect is much more sensitive to the onset of breakdown of the tori than is the EBK quantisation condition, which determines the position of the avoided crossing.

Theoretical analysis of the tunnelling problem (Wilkinson 1986) shows that the tunnelling action diverges logarithmically as the perturbation parameter $\varepsilon$ tends to zero. It turns out to be impossible to calculate the prefactor, $C$, of this logarithmic dependence in the framework of tunnelling theory, however. The fact that $S$ diverges logarithmically shows that $\Delta E$ has a power law dependence on $\varepsilon$. It is found that the exponent $\nu=C / \hbar$ usually takes values very close to integers, and a rule was given for determining these integers. The rule for determining $\nu$ can be explained by a perturbation theory, closely related to that used to calculate band gaps in the nearly-free electron limit in solid state physics. This is described in the appendix.

\section{Acknowledgments}

I am grateful to Professor M C Cross for providing computer facilities, which were funded by the US National Science Foundation. I also acknowledge financial support from a Weingart Fellowship of the California Institute of Technology. This work was not supported by any military agency.

\section{Appendix}

This appendix explains the origin of the rule given in $\$ 3.2$ for determining the integer exponents $\nu$ using perturbation theory. As it stands, this is just a heuristic argument, and is not intended to be rigorous.

The classical Hamiltonian $H\left(s_{1}, s_{2}\right)$ can be expressed in action-angle variables:

$$
H(\boldsymbol{I}, \boldsymbol{\theta})=H_{0}(\boldsymbol{I})+\varepsilon H_{1}(\boldsymbol{I}, \boldsymbol{\theta})
$$


where the expressions for $H_{0}(\boldsymbol{I})$ and $H_{1}(\boldsymbol{I}, \boldsymbol{\theta})$ are given by (2.8) and (2.11) or (2.12) (depending on which perturbation is used).

The Hamiltonian (A1) is now quantised by replacing $I, \boldsymbol{\theta}$ by operators $\hat{\boldsymbol{I}}, \hat{\boldsymbol{\theta}}$, satisfying $[\hat{\boldsymbol{\theta}}, \hat{\boldsymbol{I}}]=\mathrm{i} \hbar$. We use the representation

$$
\hat{\boldsymbol{\theta}} \rightarrow \boldsymbol{\theta} \quad \boldsymbol{I} \rightarrow-\mathrm{i} \hbar \partial / \partial \boldsymbol{\theta}
$$

In order to reproduce the Maslov indices of the true Hamiltonian we must require that the wavefunction $\psi\left(\theta_{J}, \theta_{M}\right)$ be periodic in $\theta_{M}$ but antiperiodic in $\theta_{J}$ :

$$
-\psi\left(\theta_{J}+2 \pi, \theta_{M}\right)=\psi\left(\theta_{J}, \theta_{M}\right)=\psi\left(\theta_{J}, \theta_{M}+2 \pi\right) .
$$

Of course, the Hamiltonian $\hat{H}=H(\hat{\boldsymbol{I}}, \hat{\boldsymbol{\theta}})$ will not be exactly equivalent to the true quantum Hamiltonian.

In this representation, the problem of determining the gaps in the avoided crossings is clearly very similar to that of determining the band gaps in the nearly-free electron problem in solid state physics. The action $I$ is a good quantum number for the unperturbed system, and because of (A2) it plays the role of the Bloch quasimomentum. The perturbation $\varepsilon H_{1}(\boldsymbol{I}, \boldsymbol{\theta})$ is a periodic function of $\boldsymbol{\theta}$, and is analogous to the periodic potential. The Fourier component of $H_{1}(I, \theta)$ with wavevector $\boldsymbol{n}$ causes Bragg scattering in action space through a vector $2 \pi n \hbar$. The band gap which opens up is of size $O\left(\varepsilon^{\nu}\right)$, where $\nu$ is the minimum number of Bragg scatterings needed to scatter a wave of momentum $I_{1}$ into one of momentum $I_{2}$. This is precisely the rule stated in $\S 3.2$ for calculating $\nu$. Note that this theory depends on the perturbation having only a finite number of non-vanishing Fourier coefficients.

\section{References}

Berry M V 1983 Chaotic Behaviour of Deterministic Systems, Les Houches Lectures XXXVI ed G Ioos, R H G Helleman and R Stora (Amsterdam: North-Holland) pp 171-271

Davis $\mathrm{M} J$ and Heller E J $1981 \mathrm{~J}$. Chem. Phys. 75 246-54

Feingold $M$ and Peres A 1983 Physica 9D 433-8

Landau L D and Lifshitz I M 1958 Quantum Mechanics (Oxford: Pergamon)

Lawton R T and Child M S 1981 Mol. Phys. 44 709-23

von Neumann J and Wigner E P 1929 Phys, Z. 30 467-70

Wilkinson M 1986 Physica 21D 431-54 